\title{
Análise estratégica do Projeto QualiSUS- Rede: contribuições para avaliação em saúde pública
}

\author{
Strategic analysis of the QualiSUS-Network Project: contributions for \\ evaluation in public health
}

Margarete Martins de Oliveira', Helena Eri Shimizu' $\mathbf{1}^{\mathbf{1}}$ Elizabeth Moreira dos Santos ${ }^{\mathbf{2}}$, Everton

Nunes da Silva ${ }^{1}$

DOI: 10.1590/0103-1104201912301

RESUMO O QualiSUS-Rede buscou qualificar a atenção e a gestão em saúde via organização das redes de atenção. O objetivo do estudo foi analisar o processo de formulação e implementação do QualiSUS-Rede relacionado à gestão. Trata-se de estudo qualitativo, de base documental. Fez-se uso da análise estratégica, considerando suas três dimensões: pertinência do problema priorizado; pertinência do objetivo e das soluções propostas; pertinência das parcerias. Aplicou-se a análise estratégica de forma hibridizada com a teoria de mudança em seus três eixos: cadeia de efeitos e seus condicionantes, modo de funcionamento e as interações entre os atores. Elaborou-se a linha do tempo do QualiSUS-Rede, identificando os eventos críticos. Como resultado, verificou-se que o QualiSUSRede obteve pertinência nas três dimensões da análise estratégica. No entanto, as evidências apontam que, em intervenções futuras, os seguintes aspectos precisam ser observados: i) a agilização dos tempos institucionais; ii) a fragilidade do modelo institucional de monitoramento e avaliação; e iii) dificuldade das instâncias envolvidas com a gestão de riscos, especialmente daqueles originários das transições governamentais. $\mathrm{O}$ uso de múltiplas estratégias metodológicas, com base na teoria da intervenção, considerando a cadeia de efeitos e causalidades, mostrou-se potente para explicitar a teoria e o modelo de gestão no QualiSUS-Rede.

PALAVRAS-CHAVE Atenção à saúde. Avaliação de programas e projetos de saúde. Banco Internacional para Reconstrução e Desenvolvimento. Análise qualitativa. Estudos de avaliação como assunto.

1 Universidade de Brasília (UnB) - Brasília (DF), Brasil. margareteoliveira500@ gmail.com

2 Fundação Oswaldo Cruz (Fiocruz), Escola Naciona de Saúde Pública Sergio Arouca (Ensp), Laboratório de Avaliação de Situações Endêmicas Regionais (Laser) - Rio de Janeiro (RJ), Brasil.

ABSTRACT QualiSUS-Network sought to qualify management and health care by means of organization of health networks. The objective of our study is to analyze the process of formulation and implementation of QualiSUSNetwork related to management. This is a qualitative study, on a documentary basis. Strategic analysis was used, by considering its three dimensions: relevance of the problem prioritized; relevance of the proposed objectives and solutions; relevance of partnerships. Strategic analysis was used in a hybrid way together with the theory of change considering its three axes: chain of results and its conditioners, operation mode and interactions among actors. A timeline of QualiSUS-Network was also developed, identifying critical events. As a result, it was verified that QualiSUS-Network achieved relevance in the three dimensions of the strategic analysis. However, the evidences point out that, in future interventions, the following barriers need to be observed: i) the streamlining of institutional times; ii) the weakness of the institutional model for monitoring and evaluation; iii) difficulty of the bodies involved with risk management, especially those originating from government transitions. The use of multiple methodological strategies, based on the intervention theory, considering the chain of outcomes and causalities, have proved to be potent in explaining the theory and management model in the QualiSUS-Network.

KEYWORDS Health care (public health). Program evaluation. International Bank for Reconstruction and Development. Qualitative analysis. Evaluation studies as topic. 


\section{Introdução}

O Projeto de Formação e Melhoria da Qualidade da Rede de Atenção à Saúde (QualiSUS-Rede) configura-se como uma proposta de intervenção na realidade do sistema de atenção à saúde no Brasil. Originou-se de um acordo de empréstimo entre o governo brasileiro e o Banco Mundial (Banco Internacional para Reconstrução e Desenvolvimento - Bird), com vistas a qualificar a gestão e o cuidado em saúde, por meio da organização das Redes de Atenção à Saúde (RAS)1. Logo, o QualiSUS-Rede é objeto desta análise, não só pelo grande montante de recursos públicos a ele destinados (US\$ 235 milhões)', mas porque se registra que as intervenções realizadas pelo Bird em países de baixa e média renda, inclusive no Brasil, reafirmam a centralidade de práticas de mercado, como sistema organizador e gestor mais eficiente das políticas públicas de saúde².

A organização de RAS, escopo principal do QualiSUS-Rede, é reconhecida internacionalmente como alternativa para superar a fragmentação dos sistemas de atenção à saúde, conforme já demonstrado por alguns países que conseguiram consolidar sistemas integrados ${ }^{2-6}$. Os sistemas fragmentados são caracterizados, entre outras questões, por um conjunto de pontos de atenção isolados que não se comunicam, ou se comunicam de forma inadequada, e que não oferecem ação contínua, longitudinal e integral à população ${ }^{7}$. Ademais, a população nem sempre se encontra adstrita, e as autoridades sanitárias têm dificuldades para efetivar a coordenação do cuidado $\mathbf{7 , 8}$.

As primeiras iniciativas de organização de redes de atenção ocorreram, desde os anos 1990, nos estados do Ceará, Espírito Santo e Minas Gerais, e nos municípios de Curitiba e do Rio de Janeiro ${ }^{9}$. Observadas as experiências exitosas em âmbito local e a necessidade de superar a fragmentação do sistema de atenção, em 2010, foi publicada a Portaria Ministerial GM n ${ }^{\circ}$ 4.279, que normatiza a rede de atenção à saúde no contexto do Sistema Único de Saúde (SUS), ainda que estivesse estabelecida no marco legal constitucional desde $1988^{6}$.
Concomitantemente, desde os anos 2000, as estratégias de regionalização da saúde vêm sendo implementadas visando à integração dos serviços de atenção organizados em redes de atenção à saúde 10 .

Em 2011, foram publicadas as portarias das redes temáticas: Rede Cegonha-Portaria ${ }^{\circ}$ 1.459, de 24 de junho; Rede de Urgência e Emergência Portaria ${ }^{\circ}$ 1.600, de 07 de julho; Rede de Atenção Psicossocial - Portaria ${ }^{\circ} 3.008$, de 23 de dezembro; em 2012, a Rede de Cuidados à Pessoa com Deficiência, de $n^{\circ} 793$, de 24 de abril6.

Nesse contexto, o QualiSUS-Rede caracterizou-se como a primeira estratégia formalmente adotada pelo Ministério da Saúde (MS) com o explícito objetivo de fomentar a organização das RAS, num momento em que essas se encontravam circunscritas a poucas experiências no País.

O QualiSUS-Rede se constituiu com três componentes, sendo os dois primeiros finalísticos, e o terceiro voltado para a gestão administrativa e financeira. O componente 1 visou à qualificação da gestão e do cuidado por meio da organização de redes de atenção à saúde, a partir das necessidades locais, por meio da implementação de 15 subprojetos regionais, sob responsabilidade dos estados selecionados. Desses 15 subprojetos, 10 estavam vinculados às regiões metropolitanas: Rio de Janeiro (RJ), Recife (PE), Belo Horizonte (MG), Curitiba (PR), Florianópolis (SC), Porto Alegre (RS), ABC (SP), Teresina (PI), Belém (PA), Região Integrada de Desenvolvimento - Ride (DF/GO/MG). Os outros cinco, vinculados às regiões que apresentam características sociais e geográficas singulares, denominadas Regiões Tipo. Essas incluíam: Alto Solimões (AM), região amazônica, com destacada presença indígena; Dourados/Ponta Porã (MS), região de fronteira internacional; Peba (PE/BA), região de fronteira de desenvolvimento agrícola; Topama (TO/PA/MA), região interestadual; e Cariri (CE), região com clima semiárido. As Regiões Tipo também são reconhecidas como regiões onde prevalecem as doenças negligenciadas, têm baixa cobertura de ações e de serviços, dificuldade de fixação de profissionais e baixo índice de desenvolvimento humano . 
O componente 2, operacionalizado de forma centralizada pelo MS, buscou implementar intervenções sistêmicas estratégicas, centradas em prioridades nacionais. O propósito foi o desenvolvimento de estratégias, instrumentos e sistemas de apoio que pudessem apoiar transversalmente a implantação ou o fortalecimento das RAS'

O componente 3 , de gestão, também de responsabilidade do MS, respondeu pelos processos políticos, técnicos e administrativos inerentes ao projeto QualisUS-Rede como um todo'.

Acredita-se que entender os fatores intervenientes e as características que foram peculiares ao modelo de gestão adotado possa contribuir para o aprimoramento da gestão pública de políticas de saúde ${ }^{11} \mathrm{e}$ de projetos de intervenção da mesma natureza.

A análise do processo de formulação e de implementação do QualiSUS-Rede é importante pelo seu potencial de esclarecer o caminho que a intervenção percorreu e orientar futuras decisões com vistas ao aprimoramento da gestão pública, levando-se em conta as prioridades existentes, os recursos disponíveis, os processos e os resultados a serem atingidos ${ }^{12,13}$.

De igual forma, é uma oportunidade para se identificar problemas existentes na estrutura de incentivos, os quais podem explicar grande parte das dificuldades dos gestores em alcançar os resultados pretendidos, mesmo que tenham atendido a todos os requisitos estabelecidos ${ }^{\mathbf{1 4}}$.

Estudos de análise estratégica são escassos na literatura, embora tenham potencial para auxiliar os gestores na tomada de decisão ${ }^{\mathbf{1 5}, 16}$. São mais raros ainda aqueles que exploram, para além do significado da palavra 'pertinência' no senso comum, as suas relações com a Teoria da Mudança (TdM).

Este artigo tem como objetivo realizar a Análise Estratégica (AE) da formulação e da implementação do QualiSUS-Rede, com ênfase no componente de gestão. Com a AE, buscou-se analisar, a partir das 3 dimensões que a compõem, a pertinência do projeto em termos de: i) priorização do problema a ser enfrentado (AE1); ii) objetivos e soluções propostos para combater o problema priorizado (AE2); iii) parcerias estabelecidas para atingir os objetivos (AE3) $)^{1115}$.

A articulação da análise estratégica com a teoria da mudança se deu por meio de três eixos analíticos, a cadeia de efeitos principais e aquelas relacionadas aos seus condicionantes: a de funcionamento e a das relações ${ }^{17-19}$. Por meio dessa hibridização, pretende-se desvendar a teoria subjacente ao QualiSUS-Rede e identificar o caminho percorrido para que as atividades levassem aos resultados esperados. Isto é, o caminho causal das atividades aos resultados planejados, que mostram por que e sob quais condições espera-se que os vários elos da via causal funcionem ${ }^{19}$. Além disso, a hibridização crítica com diferentes abordagens e modelos possibilita melhor apropriação das complexidades do real20.

Nesse contexto, foram metodologicamente construídas: i) a teoria da mudança, que permitiu compreender os elos de ligação causais existentes entre os principais efeitos esperados pelo QualiSUS-Rede;ii) a teoria do funcionamento, que identificou as diretrizes técnicas, os normativos e os instrumentos legais utilizados para viabilizar as mudanças pretendidas; e iii) a teoria das relações, que possibilitou identificar as parcerias estabelecidas por meio do arranjo de gestão instituído. Em cada eixo, foram identificados eventos críticos que, em alguma medida, afetaram a rota de mudança almejada pelo QualiSUS-Rede.

\section{Métodos}

Trata-se de um estudo de caso, do tipo ex post, de base documental, com uso de técnicas e de evidências qualitativas. Foram analisados dados do período de 2006 a 2016. Compreendeu quatro etapas: i) análise documental; ii) modelização do componente de gestão da intervenção, com caracterização da teoria de mudança que lhe é subjacente19; iii) elaboração de linha do tempo para identificação de eventos críticos ${ }^{21}$; e iv) elaboração da roda de análise estratégica' ${ }^{15}$.

A análise documental contemplou a revisão dos documentos referentes ao projeto, publicados ou de circulação restrita, no acervo 
do Departamento de Economia da Saúde, Investimento e Desenvolvimento (Desid) da Secretaria Executiva (SE). Com relação aos documentos de circulação restrita - tais como termos de compromisso estabelecidos entre o MS e as secretarias estaduais de saúde e os relatórios de monitoramento e de prestação de contas produzidos pela unidade gestora do projeto e pelas secretarias estatuais de saúde -, foi solicitado acesso ao Desid, que o concedeu por meio de manifestação de concordância de uso. Entre os documentos oficiais publicados, foram analisados: i) documento base de avaliação do projeto ${ }^{22}$; ii) o Contrato de Empréstimo $\mathrm{n}^{0}$ 7.632/2009, estabelecido entre o governo brasileiro e o Banco Mundial23; iii) definição da instituição vinculada e termo de cooperação técnica com a Fiocruz; iv) Portarias, que instituíram as instâncias gestoras em nível nacional e local e as diretrizes técnicas e operacionais para execução do projeto ${ }^{24-27}$; vi) definição dos quinze subprojetos regionais e dos recursos financeiros correspondentes ${ }^{27}$; e vii) relatório de avaliação do Bird ${ }^{\mathbf{2 8}}$.

A apreciação documental se deu por meio da análise de conteúdo ${ }^{29,30}$. Para a interpretação temática, apreenderam-se os núcleos de texto significantes neles contidos.

A análise documental possibilitou a identificação dos elementos que são inerentes ao QualiSUSRede, tais como a normatividade, o objetivo, o contexto, os componentes operacionais, $\mathrm{o}$ arranjo de gestão e os atores humanos, institucionais e tecnológicos. O produto dessa análise orientou a elaboração do modelo da teoria da mudança, da linha do tempo e da roda da análise estratégica.

A elaboração do modelo da teoria da mudança, a partir da cadeia de efeitos esperados, objetivou descrever a teoria subjacente ao QualiSUS-Rede. Buscou evidenciar os elos de conexão entre o problema que originou a intervenção, as estratégias, as tecnologias, as ações adotadas para superá-los e os atores envolvidos no processo, em contexto. A cadeia de efeitos esperados (TdM), usualmente expressa por diagramas, mostra o sequenciamento lógico das atividades ou dos processos da intervenção que presumidamente levariam a eles. Inclui as relações entre os processos descritos; os riscos de ruptura de cada ligação na cadeia; e a caracterização de outros fatores explicativos (explicações rivais) para a mudança esperada ${ }^{19}$. Embora representada linearmente, representa múltiplas interações entre eventos complexos, imbricados e dinâmicos ${ }^{31}$.

A elaboração da linha do tempo se deu numa perspectiva histórica, compreendendo o processo de formulação e de implementação, impresso pelo modelo de gestão adotado, desde o primeiro documento oficial de instituição do QualiSUS-Rede até os relatórios finais de prestação de contas. O objetivo desse movimento metodológico foi apreciar o contexto, identificar possíveis eventos críticos que resultaram em intensificação, atrasos e interrupções na implementação, para, com isso, evidenciar as lacunas ocorridas entre um efeito esperado e outro.

Qualificam-se como eventos críticos aqueles que por sua ocorrência geram consequências e levam à reconfiguração da intervenção ${ }^{\mathbf{2 1}}$. Compreende-se como naturalmente presentes nesse processo controvérsias que surgem a partir de diferenças existentes entre as posições dos vários atores envolvidos, pontos de vista, projetos sociais e políticos de cada um ou qualquer outra questão que esteja conjunturalmente em jogo ${ }^{16,21,32}$. Pressupõe-se que os eventos críticos materializem controvérsias atinentes ao processo de formulação e de implementação.

O modelo da análise estratégica é expresso por meio da figura da roda da análise estratégica, onde estão contidas as 3 dimensões que a compõem e que movem a roda. A elaboração da roda se deu com base em elementos contextuais datados ${ }^{15}$, identificados nos documentos normativos da intervenção em análise. Desta forma, as respostas às questões centrais fundam-se na exploração concomitante de fatores contextuais, situados historicamente, que permitiram a síntese avaliativa. A síntese avaliativa (lições aprendidas) teve como objetivo sistematizar hipóteses explicativas plausíveis às questões colocadas e reúne a análise estratégica fundada na teoria da mudança.

Este estudo atendeu à Resolução no 466/12, artigo XIII.3, e à Resolução no ${ }^{0}$ 10, de 7/4/2016, do Conselho Nacional de Saúde (CNS). 


\section{Resultados}

Os resultados são apresentados com base em três conjuntos de informações. O modelo da teoria da mudança, a linha do tempo, com identificação dos eventos críticos, e a roda da análise estratégica.

O modelo da teoria da mudança (figura 1) apresenta no eixo central da figura a cadeia de efeitos resultantes dos eventos sucessivos ocorridos no processo de formulação e de implementação do QualiSUS-Rede. As relações de condicionamento desses efeitos são representadas nos dois eixos laterais da figura, que, de forma sintetizada, descrevem as atividades que foram os requisitos condicionantes à sucessão dos resultados na cadeia de efeitos (necessários e suficientes).

Figura 1. Modelo de Teoria da Mudança do Projeto de Formação e Melhoria da Qualidade da Rede de Atenção à Saúde QualiSUS-Rede, 2019

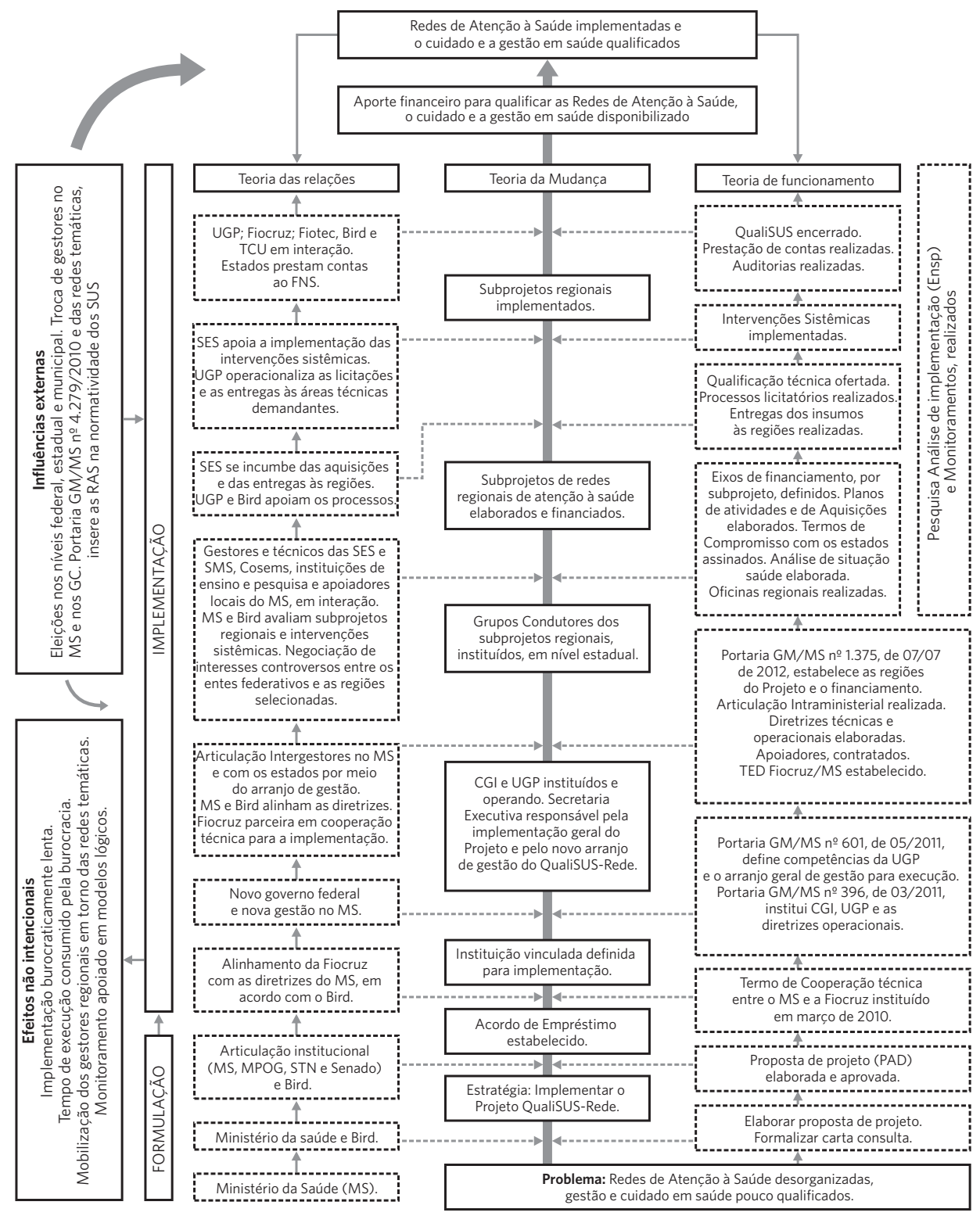

Fonte: Elaboração própria, 2019 
No eixo lateral direito, estão representados os pressupostos (a teoria de funcionamento), isto é, quais são, onde e quando os dispositivos e as ações técnicas da intervenção foram mobilizados para que o efeito subsequente na cadeia acontecesse.

No eixo lateral esquerdo estão representadas as interações entre os atores-chave que atuaram na intervenção e explicitam a teoria das relações formalizadas. Esses atores estão diretamente ligados aos dispositivos e às ações técnicas, citados na teoria de funcionamento, que, quando em interação convergente, conduzem à produção sucessiva de cada efeito.

$\mathrm{Na}$ etapa de formulação do QualiSUSRede, liderada pelo MS e pelo Bird, foi definido o problema a ser enfrentado e negociado com a Secretaria de Assuntos Internacionais (Seain), do Ministério da Economia, Planejamento, Desenvolvimento e Gestão (MPOG), e a solicitação de empréstimo ao Bird, para prover os recursos necessários à execução do projeto.

A aprovação da proposta se deu de forma articulada com outras instituições federais, tais como o Senado Federal, para aprovação do acordo de empréstimo no Congresso. Após a assinatura do acordo de empréstimo, deliberou-se que o projeto seria desenvolvido pela Fundação Oswaldo Cruz (Fiocruz), instituição vinculada ao MS. Cerca de um ano depois, o MS, pós-eleições presidenciais e sob nova gestão, retomou para si a execução do projeto e propôs um novo arranjo de gestão para a implementação, tendo o Desid como coordenador do processo ${ }^{33}$.

O arranjo formalizou as seguintes instâncias gestoras: i) Comitê Geral de Implementação (CGI), composto por representantes de todas as secretarias do MS, do Conselho Nacional dos Secretários de Saúde (Conass), Conselho Nacional de Secretarias Municipais de Saúde (Conasems), Agência Nacional de Saúde Suplementar (ANS) e Agência Nacional de Vigilância Sanitária (Anvisa), cuja atribuição foi estabelecer as prioridades de investimento, as normativas, as diretrizes técnicas e políticas e a seleção das regiões participantes; e ii) Unidade Gestora do Projeto (UGP), responsável pela coordenação geral (administrativa e financeira) do Projeto. A UGP foi composta por uma coordenação geral, um responsável por cada um dos componentes e por especialistas contratados. Para a implementação técnica das ações definidas no CGI, a UGP contava com o apoio técnico das demais secretarias do $\mathrm{MS}^{\mathbf{2 5}, \mathbf{3 3}}$.

O CGI e a UGP intensificaram a articulação com os estados, encarregados de propor os subprojetos regionais. Foram criados os Grupos Condutores (GC), que viabilizaram a identificação das demandas locais, a formulação, implementação e o monitoramento dos subprojetos regionais, sob aprovação das Comissões Intergestores Regionais (CIR). A composição dos GC deveria incluir, no mínimo, três representantes da SES, um dos municípios envolvidos, um do Conselho de Secretários Municipais de Saúde de Goiás (Cosems), um apoiador local do MS e, quando necessário, instituições de ensino e pesquisa. Identificou-se que a composição do GC variou em número, de um para outro ${ }^{34}$.

O financiamento dos subprojetos regionais alinhou-se às diretrizes estratégicas estabelecidas pelo MS para o período de 2011 a 2014, no que diz respeito à qualificação do cuidado e da gestão em saúde. Cinco eixos de financiamento orientaram a elaboração dos subprojetos e dos respectivos planos de aquisições: 1 Atenção Primária à Saúde; 2 - Redes Temáticas de Atenção à Saúde; 3 - Sistemas de Apoio Diagnóstico e Terapêutico (SADT); 4 - Sistema de Apoio Logístico; e 5 - Fortalecimento da Governança Regional. Os 3 últimos compõem a estrutura operacional da rede de atenção. Após aprovação dos subprojetos, pelo MS e pelo Bird, os grupos condutores deveriam elaborar os respectivos planos de aquisições, para manifestação de não objeção pelo $\operatorname{Bird}^{\mathbf{3 4}}$. O processo de análise ocorreu entre os meses de março e dezembro de 2012. 
Por meio do componente 1 , foram financiados quinze subprojetos regionais. Identificou-se que todos os subprojetos contemplaram nos planos de trabalho pelo menos 3 dos 5 eixos de financiamento, sendo que a maioria investiu nos 5 .

Com relação ao componente 2 , o relatório final do projeto valorizou quatro intervenções sistêmicas: curso de formação de apoiadores temáticos em ambiência na saúde; qualificação da gestão de tecnologias médico-hospitalares nas RAS, com o objetivo de mapear a capacidade de gestão dos equipamentos médico-hospitalares com foco nas Redes Temáticas Cegonha e Urgência e Emergência; qualificação da gestão da assistência farmacêutica nas RAS e implementação tecnológica do e-SUS-AB. Havia uma previsão de financiamento de um conjunto de tipos de estudos para apoiar a implementação do projeto. Apesar da iniciativa de contratação de 5 estudos, nenhuma delas foi concluída ${ }^{28}$.

A figura 1 indica algumas influências externas e efeitos não intencionais que influenciaram o projeto, tais como a burocracia em torno das regras de licitação nacionais e do Bird e a limitada capacidade técnica local para lidar com os processos de aquisição; a descontinuidade nas equipes, a extinção do Departamento de Articulação das Redes de Atenção à Saúde (Daras/MS), a publicação da Portaria GM/MS no 4.279/201035 e o Decreto Federal n ${ }^{0} 7.508$, de 28 de junho de $2011^{36}$.

A linha do tempo do QualiSUS-Rede (figura 2) apresenta de forma destacada, sombreados, alguns eventos críticos que contribuíram para a alteração de rotas na gestão do projeto. 
Figura 2. Linha do tempo do processo de formulação e implementação do Projeto de Formação e Melhoria da Qualidade da Rede de Atenção à Saúde QualiSUS-Rede (Brasil, 2006-2016)

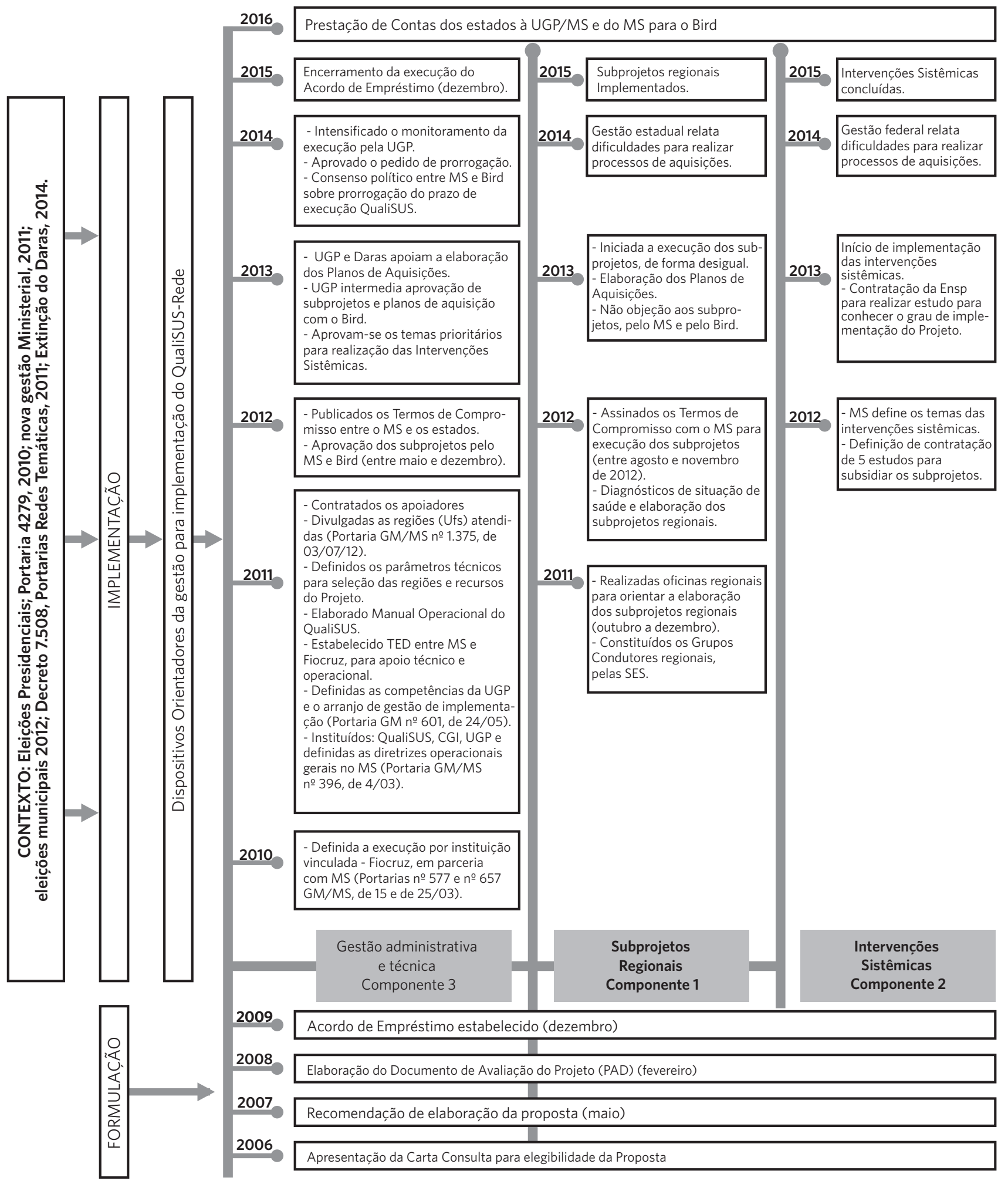


O primeiro evento crítico identificado foi o prazo para execução do projeto, o qual estava previsto para dez anos, contemplando duas fases. O recurso financeiro planejado para a primeira fase foi de US $\$ 235$ milhões, e para a segunda, de US\$ 265 milhões, oriundos de acordo de empréstimo com o Bird. A contrapartida do governo federal se daria por meio das políticas vigentes, sem incremento obrigatório de recursos financeiros novos. No entanto, após a assinatura do acordo de empréstimo, o prazo de execução foi limitado a quatro anos e seis meses, condensando-se as duas fases previstas em uma, com recursos financeiros totais no valor do estabelecido para a primeira fase. Outro evento crítico foi o fato de que a instituição vinculada designada para execução em 2010 não movimentou o projeto em 2010. Esse fato atrasou a execução do projeto em pelo menos um ano.

Houve mudança na rota da gestão do QualiSUS-Rede quando o CGI definiu os parâmetros técnicos para a seleção das regiões e recursos para os subprojetos em 2011. Observou-se nesse momento que o documento de avaliação do projeto previu que a seleção dos subprojetos contemplaria até quinze unidades federadas e que se dariam por livre concorrência, sendo qualquer estado elegível para submeter projetos a apreciação. No entanto, o CGI optou por estabelecer parâmetros de inclusão que por si definiram os estados que seriam convidados a apresentar os subprojetos regionais ${ }^{34}$. Ainda que se reconheça que os critérios foram abrangentes, isso reduziu o escopo da concorrência. Destaque-se que o tempo decorrido para a formulação da intervenção, entre a elaboração da carta consulta e a assinatura do acordo, foi de 3 anos e 10 meses.

Pode-se afirmar que a implementação do QualiSUS-Rede deriva de um processo longo de negociação entre o governo brasileiro e o Bird. Em 2014, registrava-se baixa execução financeira dos orçamentos disponíveis para os dois componentes do projeto. Diante desse cenário crítico, houve consenso entre o MS e o Bird sobre a necessidade de se prorrogar o prazo de execução do QualiSUS-Rede. Assim, o projeto foi prorrogado em 16 meses, redefinindo-se o encerramento para dezembro de $2015^{\mathbf{2 8}}$.

A roda da análise estratégica (figura 3) descreve os elementos que a compõem, considerando-se as três dimensões que lhe são inerentes. A primeira dimensão de análise (AE1) verificou a pertinência do problema selecionado, frente ao conjunto de problemas listados, pelos gestores. Os principais problemas foram: uso incipiente de mecanismos e instrumentos de gestão (planejamento, monitoramento e avaliação); financiamento público insuficiente e fragmentado; redes de atenção à saúde desorganizadas e cuidado e gestão em saúde desqualificados; e reduzida capacidade da gestão em prover conhecimento técnico para melhoria da qualidade da atenção. Entre os problemas listados, os gestores federais optaram por intervir na qualificação do cuidado e da gestão em saúde, por meio da organização de redes de atenção à saúde, tornando-o o objetivo da intervenção.

A segunda dimensão de análise (AE2) verificou se os objetivos estratégicos (soluções) propostos estariam adequados para responder às situações-problema, sendo elas: fortalecer e incorporar o uso dos mecanismos e instrumentos de gestão governamental e organizacional; financiar experiências de organização de redes e arranjos de gestão; estabelecer diretrizes técnicas e operacionais para implementação das redes e para a qualificação do cuidado; identificar atores estratégicos, problemas e prioridades locais; e produzir conhecimento para melhoria da qualidade da atenção. Sua importância está relacionada à possiblidade de se verificar a racionalidade causal da intervenção no contexto.

A terceira dimensão (AE3) verificou a pertinência dos atores envolvidos e as relações e conexões existente entre eles, assim como aprofundou a discussão sobre as respectivas atuações para lidar com as limitações administrativas e políticas inerentes ao processo de implementação. Percebeu-se que, teoricamente, a participação se deu de forma ampla, seja 
no nível federal, nos níveis estadual e local, conforme apresentado no modelo de teoria de mudança, descrito na teoria de funcionamento e na linha do tempo, mas não se identificou na documentação a participação de instituições da sociedade civil.

Figura 3. Roda da Análise Estratégica da implementação do Projeto de Formação e Melhoria da Qualidade da Rede de Atenção à Saúde - QualiSUS-Rede, 2019

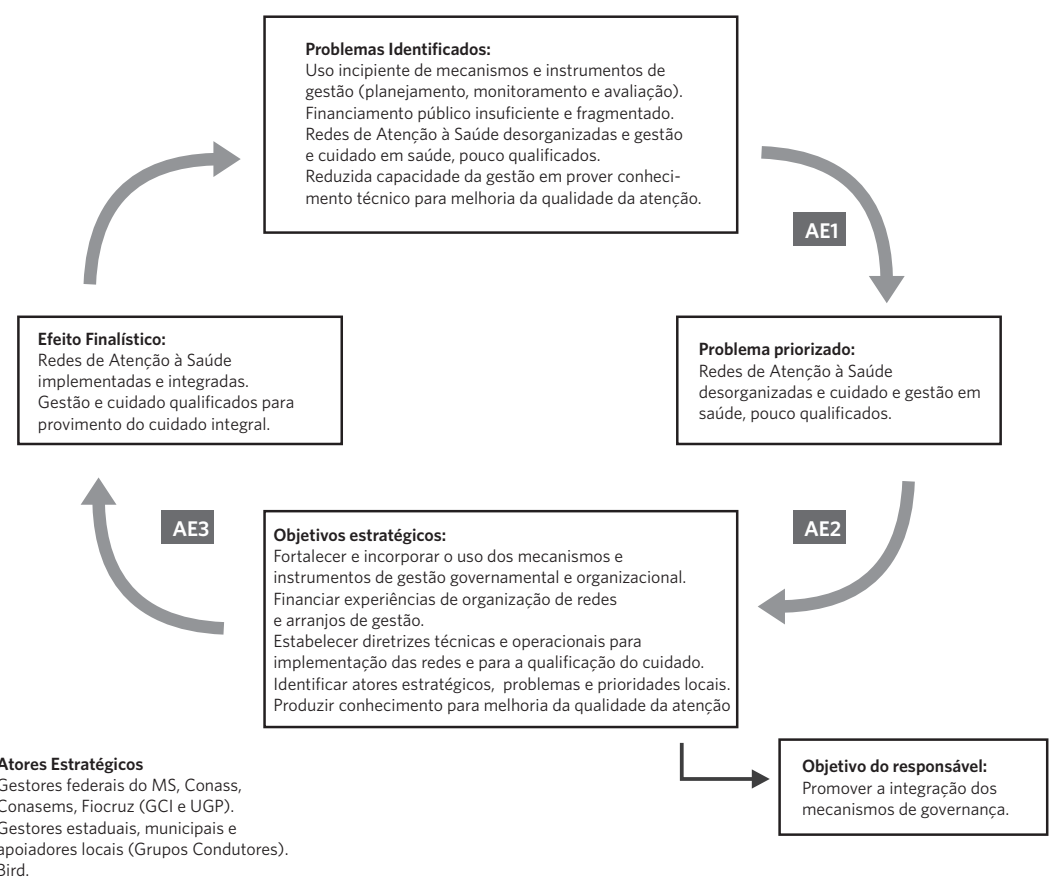

Fonte: Adaptado de Champagne F, et al.11

\section{Discussões}

A teoria de mudança e a identificação dos eventos críticos por meio da organização cronológica das ações do QualiSUS-Rede, registradas na linha do tempo, desvelaram o modelo de gestão adotado e iluminaram o processo de formulação, favorecendo o conhecimento das ações implementadas. Essas duas abordagens permitiram a identificação dos condicionantes da estratégia QualiSUS-Rede de forma a embasar a exploração empírica das dimensões da AE. Os três eixos não devem e não foram interpretados como paralelos, pois são conjuntos de pressupostos a serem compreendidos de forma interativa ${ }^{19,31,37}$. Em outras palavras, os eixos laterais são condições para a ocorrência dos efeitos do eixo central, expressam as condições 'se' e 'então', do inglês 'if' and 'then'. Neste artigo, esse é o norteador da discussão.

Observou-se que a formulação do projeto QualiSUS-Rede contou com a participação de gestores que atuavam no MS e no Banco Mundial, cujas relações geraram um modelo de gestão que não incluiu outros atores estratégicos, na fase de formulação do projeto.

A análise estratégica evidenciou que a 
escolha do problema foi bem-sucedida (AE1). Primeiro, a necessidade de organização das RAS não é tema novo no Brasil, e as evidências sugerem que a organização das RAS é problemática no contexto do SUS. As estratégias de organização das RAS adotadas no Brasil se apoiam em experiências de outros países que construíram sistemas universais de saúde ${ }^{38}$. O desenho da federação brasileira e as dificuldades de articulação com as quais os vários municípios de uma mesma região se defrontam quando procuram promover a integração e a hierarquização dos serviços de saúde, em especial, da rede de atenção básica com os níveis mais altos de complexidade, são alguns desses problemas ${ }^{39-41}$.

A inclusão de atores dos três níveis de gestão só ocorreu no processo de implementação quando todas as diretrizes técnicas, de financiamento e normativas já estavam definidas pelo nível central, e não inclui atores do controle social. Revisões sistemáticas realizadas sobre a transformação de grandes sistemas de saúde advogam que a participação de todos os atores interessados é condição essencial quando se propõem a modelar o sistema de saúde, em torno de objetivos comuns ${ }^{\mathbf{1 0 , 4 2}}$.

Quanto à apreciação de adequação e conformidade das escolhas de ações e estratégias adotadas (AE2), pode-se afirmar que investir na estrutura operacional da rede, por meio dos subprojetos regionais, configurou-se como uma opção alinhada à perspectiva de sustentabilidade da implementação da RAS e accountability mútua ${ }^{\mathbf{4 3}}$, portanto, uma escolha adequada. Afirma-se que a falta de entendimento sobre a totalidade da estrutura operacional (dispositivos de funcionamento) tem sido responsável por problemas de implantação das redes, justificada pela pouca atenção dada à organização dos sistemas de apoio, logísticos e de governança44.

Os subprojetos elaborados, compostos por muitos dos eixos temáticos disponibilizados para financiamento, mostraram que o processo de priorização técnica para definir-se o investimento necessário para atender às diversas necessidades inerentes à organização das redes de atenção levou os gestores a não observarem a existência de suficiência e a otimização dos recursos financeiros com base nos objetivos comuns, mas nas necessidades individuais dos municípios. Deve-se considerar que a implementação de uma política se dá com muitas incertezas, uma vez que o envolvimento de múltiplos atores, geralmente com concepções e interesses próprios, e não necessariamente coincidentes, pode levar a ambientes de disputas e dificuldades de centralidade em torno de objetivos comuns ${ }^{\mathbf{1 4}}$.

Além disso, dar operacionalidade e concretude à execução dos subprojetos regionais num contexto de desmobilização dos grupos de trabalho locais, causados pelos diferentes períodos eleitorais e de mudanças governamentais, imprimiu morosidade na execução do projeto, ainda que os grupos condutores, com apoio dos apoiadores locais que atuaram como interlocutores entre os estados e o MS, tenham feito seu papel de dinamizar as agendas e se dedicado a manter regularidade na execução $0^{\mathbf{4 5}, 46}$.

A necessidade de fazer convergirem as regras de licitação existentes no Brasil com as regras estabelecidas pelo Bird para aquisição de bens e serviços foi um fator de dificuldade e agregou demora na consecução das aquisições. A administração pública tem problemas nesse setor. Ainda há carência de profissionais para atuarem nessa área, e a instrumentalização desses processos é precária, comprometendo-se a fase mais importante da licitação, que é a de planejamento do certame, que se relaciona com: especificação, quantificação, pesquisa de mercado, estimativa de preço, elaboração do projeto básico ou do termo de referência ${ }^{\mathbf{4 7}, \mathbf{4 8}}$.

Identificaram-se múltiplas tentativas de contratação dos estudos sistêmicos catalizadores da implementação referente ao componente 2 . Atender à modalidade de contratação praticada pelo Bird para essa ação, em função do tempo disponível para realizá-la, mostrou-se inadequado. A duração, em torno de 24 meses do início à conclusão do processo, inviabilizou a realização dos estudos previstos. 
Com relação à terceira dimensão que compõe a Análise Estratégica (AE3), exceto na formulação, verificou-se a pertinência das parcerias estabelecidas. No nível federal, todas as secretarias do MS compuseram o arranjo de gestão. Já no nível estatual, os grupos condutores foram compostos por representantes das secretarias estaduais e municipais de saúde, dos Cosems e de universidades ${ }^{1}$.

Percebeu-se, ao analisar as atas de reunião da UGP, que houve desmobilização do CGI no decorrer do terceiro ano de execução. Isso não se explica apenas pelo esvaziamento de suas competências político-estratégicas, à medida que o projeto progredia no processo de sua execução, mas pelo fato de que a execução incipiente tornou invisível a necessidade de acompanhamento por parte desses atores. A adoção de uma política não necessariamente garante a sua implementação, que pode encontrar resistência, ser cooptada ou transformada, muitas vezes tornando-se sem significado em face dos muitos atores e interesses que a compõem ${ }^{\mathbf{1 4}}$.

Toda indução financeira gera ritos formais e não formais. O rito formal está explícito no modelo normativo operacional adotado, que por si só engendra arranjos contextuais que se corporificam como arranjos não formais ou tácitos, através dos mecanismos e processos de implementação. A indução catalizadora não se implementa linearmente, mas ocorre submersa em contextos nos quais fatores complicadores constantemente requerem negociações, ajustes internos e mesmo desvios do objetivo proposto.

\section{Considerações finais}

Este estudo viabilizou o conhecimento do caminho percorrido pelo Qualisus-Rede, da formulação à implementação, com seus resultados esperados, expressos nos modelos elaborados. Ao desvelar a cadeia de causalidade explicitada nos normativos que orientaram a implementação da intervenção, identificaram-se as hipóteses de mudança esperadas pelo QualiSUS-Rede. Os modelos se mostraram potentes para identificar todos os atores envolvidos e suas respectivas ligações com a teoria de funcionamento para produção dos efeitos de causalidade.

As evidências apontaram três aspectos fundamentais para o aprimoramento da gestão do QualiSUS-Rede: i) a agilização dos tempos institucionais; ii) a fragilidade do modelo institucional de monitoramento e avaliação da estratégia; e iii) dificuldade das instâncias internas do projeto com a gestão de riscos, especialmente daqueles originários dos períodos de transição de governo. Todos se relacionam à prospecção de contexto, análise dos riscos e pactuação da hierarquia de valor agregado, inerentes à apreciação ex ante da estratégia e relacionados à sua modelização.

A análise estratégica evidenciou que os arranjos de gestão não se formam apenas em espaços formais de negociação, principalmente no âmbito regional/local. O fato incontestável é que, nesse âmbito, os arranjos não se sustentam e se descontinuam. Assim, parece recomendável a exploração desses, por estudos que utilizem a teoria de mudança, esquadrinhem de maneira explícita a racionalidade causal da estratégia adotada. Mais significativa ainda parece ser a necessidade de, no momento de formulação, desenhar não só o modo de funcionamento técnico, mas antecipar as relações entre os atores e seus interesses coletivos e/ou privados que tenham prevalecido nas negociações e nos acordos. $\mathrm{Da}$ mesma forma, são requeridos estudos sobre modelos de monitoramento capazes de informar a ação, prática ainda incipiente na implementação de políticas públicas no Brasil.

No processo de mobilização de teorias que tornassem explícitos os pressupostos que informaram o QualiSUS-Rede, a utilização da teoria da mudança e de seus componentes lógicos (racionalidade causal, teoria de funcionamento e teoria das relações) para a modelização das etapas da análise estratégica tornou a construção de evidências transparente, possibilitando a validação por pares e viabilizando devolutivas úteis para o aprimoramento institucional. 
O estudo demonstrou potência de se trabalhar com múltiplas estratégias metodológicas, com base na teoria da intervenção, considerando a cadeia de efeitos e causalidades que compuseram a intervenção. $\mathrm{O}$ estudo aqui apresentado, aplicado ao QualiSUS-Rede, configura-se, no entendimento dos autores, um caminho para teorização de outras intervenções de mesma natureza, considerando naturalmente as especificidades e os contextos em que cada um se insere.

\section{Referências}

1. Brasil. Ministério da Saúde. Manual Operacional do Projeto QualiSUS-rede -Documento Base - Volume 1 [internet]. Brasília, DF: Ministério da Saúde; 2010. 56 p. [acesso em 8 jan 2018]. Disponível em: http:// portalarquivos.saude.gov.br/images/pdf/2014/outubro/08/VoliDocBaseQualisUS-RedeWeb.pdf.

2. Organização Pan-americana da Saúde; Organização Mundial da Saúde. Renovação da Atenção Primária em Saúde nas Américas: Documento de Posicionamento da Organização Pan-Americana da Saúde/ Organização Mundial da Saúde (OPAS/OMS). Washington, USA: OPAS/OMS; 2007.

3. Mendes EV. Revisão bibliográfica sobre redes de atenção à saúde [internet]. Minas Gerais: Secretaria de Estado de Saúde de Minas Gerais; 2007. 154 p. [acesso em 2 fev 2019]. Disponível em: http://bvsms.saude. gov.br/bvs/publicacoes/revisao_bibliografica_redes. pdf.

\section{Colaboradores}

Oliveira MM (0000-0002-3926-4519)*, Santos EM (0000-0002-2137-7048)* e Silva EN (0000-0001-8747-4185)* contribuíram substancialmente para a concepção e o planejamento, a análise e interpretação dos dados, da revisão crítica do conteúdo e da aprovação da versão final. Shimizu HE (0000-0001-5612$5695) *$ contribuiu para a concepção, orientação, e revisão da versão final.

4. Mendes EV. As Redes de Atenção à Saúde [internet]. 2. ed. Brasília, DF: Organização Pan-America da Saúde; 2011. 549 p. [acesso em 21 dez 2017]. Disponível em: http://www.saude.sp.gov.br/resources/ses/perfil/gestor/documentos-de-planejamento-em-saude/ elaboracao-do-plano-estadual-de-saude-2010-2015/ textos-de-apoios/redes_de_atencao_mendes_2.pdf.

5. Shimizu HE. Percepção dos gestores do Sistema Único de Saúde acerca dos desafios da formação das Redes de Atenção à Saúde no Brasil. Physis (Rio J.). [internet]. 2013 [acesso em 2018 nov 12]; 23(4):1101-1122. Disponível em: http://www.scielo.br/scielo.php?script=sci arttext\&pid=S0103-73312013000400005\&lng=en\&n $\mathrm{rm}=\mathrm{iso} \& \mathrm{tlng}=\mathrm{pt}$.

6. Magalhães Jr. HM. Redes de Atenção à Saúde: rumo à integralidade. Divulg. saúde debate. 2014; 52(15-37). and Contributor ID). 
7. Silva SF. Organização de redes regionalizadas e integradas de atenção à saúde: desafios do Sistema Único de Saúde (Brasil). Ciênc. Saúde Colet. [internet]. 2011 [acesso em 14 jan 2019]; 16(6):2753-2762. Disponível em: http://www.scielo.br/scielo.php?script=sci arttext\&pid=S1413-81232011000600014\&lng=pt\&tln $\mathrm{g}=\mathrm{pt}$.

8. Mendes EV. As redes de atenção à saúde. Ciênc. Saúde Colet. [internet]. 2010 [acesso em 2 fev 2019]; 15(5):2297-2305. Disponível em: http://www.scielo.br/scielo.php?script=sci_arttext $\&$ pid $=\mathrm{S} 1413$ $-81232010000500005 \& \operatorname{lng}=$ pt\&nrm=iso\&tlng=pt.

9. Albuquerque MV, Viana ALD. Perspectivas de região e redes na política de saúde brasileira. Saúde debate [internet]. 2015 [acesso em $2017 \mathrm{dez} 21$ ]; 39 (esp): 2838. Disponível em: http://www.scielo.br/pdf/sdeb/ v39nspe/0103-1104-sdeb-39-spe-00028.pdf.

10. Viana ALD, Bousquat A, Melo GA, et al. Regionalização e Redes de Saúde. Ciênc. Saúde Colet [internet]. 2018 [acesso em 2018 out 23]; 23(6):1791-1798. Disponível em: http://www.scielo.br/scielo.php?script=sci arttext\&pid=S1413-81232018000601791\&lng=pt\&tln $\mathrm{g}=\mathrm{pt}$.

11. Champagne F, Contandriopoulos AP, Brousselle A, et al. A Avaliação no Campo da Saúde: conceitos e métodos. In: Brousselle A, Champagne F, Contandriopoulos AP, organizadores. Avaliação: conceitos Métodos. Rio de Janeiro: Editora Fiocruz; 2013. p. 41-60.

12. Brousselle A, Champagne F, Contandriopoulos AP, et al. Avaliação conceito e métodos. Rio de Janeiro: Fiocruz; 2011. 291 p.

13. Furtado JP, Vieira-da-Silva LM, Furtado JP, et al. A avaliação de programas e serviços de saúde no Brasil enquanto espaço de saberes e práticas.

Cad. Saúde Pública (Online). [internet]. 2014 [acesso em 2018 fev 3]; 30(12):2643-2655. Disponível em: http://www.scielo.br/scielo.php?script=sci arttext\&pid=S0102-311X2014001202643\&lng=pt\&t lng=pt.
14. Arretche M. Uma contribuição para fazermos avaliações menos ingênuas. In: Barreira MCRN, Carvalho MCB, organizadores. Tendências e perspectivas na avaliação de políticas e programas sociais. São Paulo: IEE/PUC; 2001. p. 43-56.

15. Champagne F, Brousselle A, Contandriopoulos AP, et al. A Análise Estratégica. In: Brousselle A, Champagne F, Contandriopoulos AP, organizadores. Avaliação: conceitos Métodos. Rio de Janeiro: Fiocruz; 2013. p. 95-104.

16. Soares BC, Cardoso GCP, Figueiró AC. Análise estratégica da Vigilância Epidemiológica em tuberculose: uma experiência local. Saúde debate [internet]. 2017 [acesso em 2018 mar 11]; 41(esp):22-33. Disponível em: http://www.scielo.br/pdf/sdeb/v4lnspe/01031104-sdeb-41-nspe-0022.pdf.

17. Chen H-T. A conceptual framework of program theory for practiotiners. In: Chen HT, organizador. Practical program evaluation: assessing and improving, planning, implementation and effectiveness. Thousand Oaks, California: SAGE; 2005. p. 15-45.

18. Rossy PH, Lipsey MW, Freeman HE. Expressing and assessing program theory. In: Rossy PH, Lipsey MW, Freeman HE, organizadores. Evaluation: a Systematic Approach a systematic approach. Thousand Oaks, California: SAGE; 2004. p. 133-68.

19. Ottawa JM. Useful Theory of Change Models. Can J Progr Eval [internet]. 2015 [acesso em 28 jul 2018]; 30(12):119-142. Disponível em: https://evaluationcanada.ca/system/files/cjpe-entries/30-2-119_0.pdf.

20. Santos EM, Oliveira EA, Cardoso G, et al. Avaliações inclusivas: uma reflexão sobre possibilidades e implicações de abordagens participativas para a prática em avaliação. Rev Salud Ambiental. 2018; 18(2):172181.

21. Figueiro AC, Araújo Oliveira SR, Hartz Z, et al. A tool for exploring the dynamics of innovative interventions for public health: the critical event card. Int $\mathrm{J}$ Public Health. 2017; 62(2):177-186. 
22. World B. Project appraisal document for a network formation and quality improvement projetct (QUALISUS-REDE) [internet]. Brasília, DF: QUALISUS-REDE; 2008. [acesso em 2018 mar 3]. Disponível em: http://documents.worldbank.org/curated/ en/566961468225310105/pdf/423590PAD0P0881E 0ONLY10R20091000211.pdf.

23. World B. Loan Agreement (Health Network Formation and Quality Improvement Project - QUALISUS-REDE) [internet]. Brasília, DF: QUALISUS-REDE; 2009. [acesso em 2019 out 4]. Disponível em: http://documents.worldbank.org/ curated/en/444211468015668781/pdf/CONFORMED0Loan11BR010Qualisus0Rede0.pdf.

24. Magalhães R. Constrangimentos e oportunidades para a implementação de iniciativas intersetoriais de promoção da saúde: um estudo de caso. Cad. Saúde Pública [internet]. 2015 [acesso em 20 jan 2019]; 31(7):1427-1436. Disponível em: http://www.scielosp.org/scielo.php?script=sci_arttext\&pid=S0102-311X2015000701427.

25. Brasil. Portaria $n^{\circ} 577$, de 15 de março de 2010. Institui o Projeto de Formação e Melhoria da Qualidade de Rede de Saúde - QualiSUS- Rede, o Comitê Gestor de Implementação - CGI, a Unidade de Gerenciamento de Projeto (UGP). [internet]. Diário Oficial da União. 16 Mar 2010. [acesso em 20 jan 2019]. Disponível em: http://bvsms.saude.gov.br/bvs/saudelegis/gm/2010/prt0577_15_03_2010_comp.html.

26. Brasil. Portaria ${ }^{\circ} 601$, de 24 de maio de 2011. Dispõe sobre a organização e as competências da Unidade de Gestão do Projeto de Formação e Melhoria da Qualidade de Re-de de Saúde (QualisUS-Rede) e define o arranjo de gestão para a execução dos subprojetos. [internet]. Diário Oficial da União. 25 Maio 2010. [acesso em 20 jan 2019]. Disponível em: http://bvsms.saude. gov.br/bvs/saudelegis/se/2011/prt0601_24_05_2011. html.

27. Brasil. Portaria $\mathrm{n}^{\circ} 1.375$, de 3 de Julho de 2012. Define as regiões selecionadas para participação e implementação das ações dos subprojetos do Projeto QualiSUS-Rede. Diário Oficial da União. 4 Jul 2012.
28. World Bank. Independent Evaluation Group (IEG). BR Health Network Formation \& Quality [internet]. Washington: World Bank; 2017. 10 p. [acesso em 2019 out 7]. Disponível em: http://documents.worldbank. org/curated/en/437261485364913871/pdf/ICRR-Disclosable-P088716-01-25-2017-1485364900963.pdf.

29. Bardin L. Análise de Conteúdo. Lisboa: Edições 70; 1977. $223 \mathrm{p}$.

30. Spink P. Análise de documentos de domínio publico. In: Spink MJ, organizador. Práticas discursivas e produção de sentidos no cotidiano:aproximação teóricas e metodológicas. São Paulo: Cortez; 2004. p. $123-51$.

31. Davis R. Scale, Complexity and the Representation of Theories of Change: Part II. Evaluation [internet]. 2005 [acesso em 10 fev 2019]; 11(1):133-149. Disponível em: http://citeseerx.ist.psu.edu/viewdoc/downl oad?doi=10.1.1.914.1955\&rep=repl\&type=pdf.

32. Clavier C, Sénéchal Y, Vibert S, et al. A theory-based model of translation practices in public health participatory research. Sociol Health Illn. 2012; 34(5):791805.

33. Brasil. Portaria ${ }^{\circ} 396$, de 4 de março de 2011. Institui o Projeto de Formação e Melhoria da Qualidade de Rede de Saúde (Quali-SUS-Rede) e suas diretrizes operacionais gerais. [internet]. Diário Oficial da União. 4 Mar 2011. [acesso em 8 jan 2018]. Disponível em: ftp://ftp.saude.sp.gov.br/ftpsessp/bibliote/ informe_eletronico/2011/iels.mar.11/Iels44/U_PT-MS-GM-396_040311.pdf.

34. Brasil. Ministério da Saúde. Manual Operacional. Volume 2. Adesão ao projeto e formuação das propostas dos subprojetos. [internet]. Brasília, DF: Ministério da Saúde; 2012. [acesso em 2019 jun 6]. Disponível em: http://portalarquivos2.saude.gov.br/images/ pdf/2014/outubro/08/Vol2AdesaoFormulaSubprojetoQualiSUS-RedeWeb.pdf.

35. Brasil. Ministério da Saúde. Portaria no 4.279, de 30 de dezembro de 2010. Estabelece diretrizes para a organização da Rede de Atenção à Saúde no âmbito 
do Sistema Único de Saúde (SUS) [internet]. Diário Oficial da União. 31 Dez 2010. [acesso em 11 jan 2019]. Disponível em: http://conselho.saude.gov.br/ultimas_ noticias/2011/img/07_jan_portaria4279_301210.pdf.

36. Brasil. Decreto $n^{0} 7.508$, de 28 de junho de 2011. Regulamenta a Lei $n^{\circ}$ 8.080, de 19 de setembro de 1990, para dispor sobre a organização do Sistema Único de Saúde - SUS, o planejamento da saúde, a assistência à saúde e a articulação interfederativa, e dá outras providências. [internet]. Diário Oficial da União. 29 Jun 2011. Disponível em: http://www.planalto.gov.br/ccivil_03/_ato2011-2014/2011/decreto/D7508.htm.

37. Patton MQ. Developmental evaluation for equity-focused evaluations. In: Marco Segone, editor. Evaluation for equitable development results [internet]. New York: UNICEF; 2012. p. 102-114. [acesso em 2019 fev 10]. Disponível em: http://www.unicef.org/evaluation/index.html.

38. Kuschnir R, Chorny AH. Redes de atenção à saúde: contextualizando o debate. Ciênc. Saúde Colet. 2010; 15(5):2307-2316.

39. Assis E, Cruz VS, Trentin EF, et al. Regionalização e novos rumos para o SUS: A experiência de um colegiado regional. Saúde e Soc. 2009; 18(supl):17-21.

40. Machado CV, Lima LD, Viana AL, et al. Federalism and health policy: the intergovernmental committees in Brazil. Rev Saude Publica. [internet]. 2014 [acesso em 20 fev 2019]; 48(4):642-650. Disponível em: http://www.scielosp.org/scielo.php?script=sci_artt ext\&pid=S0034-89102014000400642.

41. Gadelha CAG, Machado CV, Lima LD, et al. Saúde e territorialização na perspectiva do desenvolvimento. Ciênc. Saúde Colet [internet]. 2011 [acesso em 22 jan 2019]; 16(3):3003-3016. Disponível em: http://www. scielo.br/pdf/csc/vl6n6/38.pdf.

42. Best A, Greenhalgh T, Lewis S, et al. Large-System Transformation in Health Care: A Realist Review. Milbank Q [internet]. 2012 [acesso em 2019 fev 7]; 90(3):421-456. Disponível em: http://www.ncbi.nlm. nih.gov/pubmed/22985277.
43. Organization for Economic Co-operation and Development. The Paris Declaration on Aid Effectiveness and the Accra Agenda for Action [internet]. Paris: OECD; 2008. [acesso em 2019 fev 10]. Disponível em: https://www.oecd.org/dac/effectiveness/34428351. pdf.

44. Mendes EV. Comentário sobre as Redes de Atenção à Saúde no SUS. Divulg. Saúde debate. 2014; (52):3849.

45. Casanova AO, Cruz MM, Giovanella L, et al. A implementação de redes de atenção e os desafios da governança regional em saúde na Amazônia Legal: uma análise do Projeto QualisuS-Rede. Ciênc Saúde Colet. [internet]. 2017 [acesso em $2 \mathrm{fev}$ 2019]; 22(4):1209-1224. Disponível em: http://www. scielo.br/scielo.php?script=sci_arttext\&pid=S1413$-81232017002401209 \& \operatorname{lng}=$ pt\&tlng=pt.

46. Oliveira EA, Cardoso GCP, Santos EM, et al. O apoiador local como ator estratégico na implementação do QualiSUS-Rede: engenheiros de conexão? Saúde debate [internet]. 2017 [acesso em 2018 jan 7]; 41(esp):275-289. Disponível em: http://www.scielo.br/scielo.php?script=sci_arttext \&pid=S0103$-11042017000500275 \& \operatorname{lng}=$ pt\&tlng=pt.

47. Fernandes JUJ. Sistema de Registro de Preços e Pregão Presencial e Eletrônico. 6. ed. Belo Horizonte: Fórum; 2015. 636 p.

48. Martins VLS, Marques LS. A proposta de um modelo de gestão e fiscalização de contratos em uma unidade federal de saúde. Brazilian J Dev [internet]. 2018 [acesso em 2019 mar 22]; 4(5):1838-1852. Disponível em: http://www.scielo.br/scielo.php?script=sci arttext\&pid=S0104-40602000000200013\&lng=pt\& tlng=pt.

Recebido em 29/03/2019

Aprovado em 16/10/2019

Conflito de interesses: inexistente

Suporte financeiro: não houve 